\title{
Combined maximum-quantum and DOSY 3D experiments provide enhanced resolution for small molecules in mixtures
}

\author{
G. N. Manjunatha Reddy, ${ }^{a}$ Mehdi Yemloul ${ }^{b}$ and Stefano Caldarelli ${ }^{a, b_{*}}$
}

\begin{abstract}
We illustrate here as the combination of high-order maximum-quantum (MaxQ) and Diffusion-Ordered SpectroscopY (DOSY) NMR experiments in a 3D layout allows superior resolution for crowded NMR spectra. Non-uniform sampling (NUS) allows compressing the experimental time effectively to reasonable durations. Because diffusion effects were encoded within multiple-quantum coherences, increased sensitivity to magnetic field gradients is observed, requiring compensation for convection effects. The experiment was demonstrated on the spectra of a mix of small polyaromatic molecules. Specifically, in the case analyzed, the experiment provided an extreme simplification through the MaxQDOSY-MaxQ projection plane that presents one peak per molecule. Copyright $\odot 2016$ John Wiley \& Sons, Ltd.
\end{abstract}

Keywords: NMR; ${ }^{1} \mathrm{H}$; mixtures; DOSY; multiple-quantum; non-uniform sampling (NUS)

\section{Introduction}

Diffusion-Ordered SpectroscopY (DOSY) for mixtures provides a one-pot measure of molecular self-diffusion coefficients. ${ }^{[1-3]}$ These are directly related to the hydrodynamic radius; thus, in the corresponding two-dimensional diffusion ordered diagram the signals from different species are represented by their chemical shifts along one dimension and separated according proportionally to their volume along the other. ${ }^{[4-6]}$ The DOSY processing display quickly became a very popular and powerful application for the characterization of complex mixtures without the need for physical separation. ${ }^{[7,8]}$ On the other hand, it is often the case for complex mixtures that the NMR spectra are crowded, resulting in strongly overlapping patterns. This situation interferes severely with the algorithms commonly employed to calculate the diffusion coefficients, which are all approximations of the Inverse Laplace Transform, and thus very unstable with respect to multiexponential behavior, and particularly so for close values of the diffusion coefficients. One approach to tackle this problem is to increase the resolution through three-dimensional sequences. ${ }^{[9-12]}$ While the duration of such experiments can represent a serious drawback, methods for shortening significantly the acquisition time of multidimensional techniques are becoming available, ${ }^{[13-16]}$ as it will be discussed more in the following. We note in passing that an alternative approach to increase the resolution is the dispersion of the diffusion coefficients according to specific interactions with a matrix. ${ }^{[17-22]}$

Multiple-quantum NMR is another technique that has been proposed for the analysis of complex spectra, because of its ability to simplify the spectra of multicomponent samples, ${ }^{[23-32]}$ and it has been recently adapted to the characterization of complex mixtures of small molecules. The latest variant of this method involves the use of the highest coherence order in a proton-coupled spin system, the maximum-quantum (MaxQ) ${ }_{1}^{[33-36]}$ based on the fact that this coherence provides a singlet in the 'indirect' dimension correlating all peaks in the 'direct' (single-quantum) dimension of the participating spin system, giving a result similar to a DOSY diagram. ${ }^{[36]}$ MaxQ NMR was demonstrated to be useful to overcome spectral crowding in the case of very complex mixtures of small molecules, with remarkable results on natural mixtures. In general, $p Q$ NMR filters out of the spectrum signals from spin systems with a size (number of coupled spins $1 / 2$, of example) smaller than $p$. Thus this is a good building block for pulse sequences to be incorporated in a two- or three-dimensional diffusion-edited analysis to reduce the overlapping of the signals in the DOSY diagram. The theoretical general ${ }^{[37]}$ and optimal ${ }^{[38]}$ aspects of multiple-quantum NMR creation and reconversion have been discussed at length in the literature and thus in the following we shall rather focus on the aspects concerning multiple-quantum diffusion.

The diffusion measurement using multiple-quantum coherences was demonstrated in the cases of scalar, dipolar, and quadrupolar coupling. ${ }^{[39-44]}$ It has been shown that if the dephasing information resulting from translational displacements is encoded while a $p$-coherence order has been selected, the apparent gradient, or the effective magnetogyric ratio, is multiplied by $p$. In this case, the echo signal attenuation of the PGSE NMR experiment is governed by a term of the type ${ }^{[39,40]}$ :

$$
e^{\left(-\gamma_{\text {eff }}^{2} g^{2} D \delta^{2}(\Delta-\delta / 3)\right)}
$$

\footnotetext{
* Correspondence to: Stefano Caldarelli, Aix Marseille Université, Centrale Marseille, CNRS, iSm2 UMR 7313, 13397 Marseille, France. E-mail: s.caldarelli@univ-amu.fr

a Institut de Chimie des Substances Naturelles, CNRS UPR 2301 Avenue de la Terrasse 91190, Gif-sur-Yvette, France

b Aix-Marseille Université, CNRS, Centrale Marseille, Institut des Sciences
} Moleculaires de Marseille, (iSm2, UMR 7313), 13397, Marseille, France 
where, for homonuclear $p$-quantum coherence, the apparent magnetogyric ratio $\gamma_{\text {eff }}=p \gamma, \delta$ is the gradient pulse duration, $\Delta$ the diffusion delay, $g$ the field gradient intensity, and $D$ the diffusion coefficient. Equation (1) is calculated assuming square pulses for the field gradient, with other formulas available for arbitrary pulse shapes. The combination of diffusion measurements with Multiple-Quantum NMR was first proposed by Zax and Pines, ${ }^{[39]}$ and for mixture analysis by Dalvit et al. ${ }^{[45]}$ In this latter case, one- and two-dimensional diffusionweighted double-quantum experiments (DWDQ) were obtained by incorporating diffusion-editing before the $M Q$ pumping period. In this experiment, the diffusion effects were encoded with $p=1$. Recently, a similar application using a 3D version was proposed by sequentially concatenating a DWDQ and DOSY. ${ }^{[46]}$

In this work, we describe the application to mixtures analysis of $2 \mathrm{D}$ and $3 \mathrm{D}$ experiments where the diffusion is encoded on multiple-quantum coherence from homonuclear scalar couplings, up to the MaxQ order. Compensation of vertical

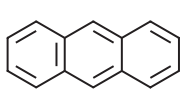

anthracene

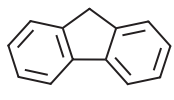

fluorene

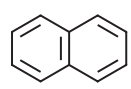

naphthalene

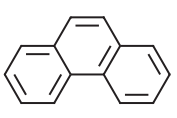

phenanthrene
Figure 1. Structure of the four polyaromatic molecules in the mixture under investigation. convection effects was integrated, ${ }^{[47]}$ more important here because of apparent amplification of the field because of $\mathrm{MQ}$ encoding, its known limitations notwithstanding. ${ }^{[48]}$ Standard non-uniform sampling (NUS) was incorporated for optimal time/resolution efficiency. ${ }^{[49]}$

\section{Experimental Methods}

All products were purchased from Sigma-Aldrich. A model mixture of four aromatic molecules (anthracene, fluorene, naphthalene, phenanthrene, Fig. 1) was prepared with a concentration of $10 \mathrm{mM}$ for each solute in deuterated solvents with $50 / 50(\mathrm{v} / \mathrm{v})$ DMSO- $d_{6} /$ acetone- $d_{6}$. These four molecules possess the same maximum quantum order (4Q), and their diffusion coefficients are close to each other. This makes this mixture particularly well suited for demonstrating the accuracy of the method and its separation capabilities.

All experiments were performed at ambient temperature on a Bruker Avance III spectrometer operating at $600 \mathrm{MHz}$, equipped with a triple resonance high-resolution probe producing gradients with a maximum strength of $60 \mathrm{G} \mathrm{cm}^{-1}$. All NMR datasets were processed in TOPSPIN 3.2 version (Bruker BioSpin, Germany). To obtain reference values of diffusion coefficients, standard DOSY spectra were recorded by a conventional pulse sequence, based on the stimulated echo and incorporated bipolar gradient pulses and an eddy-current delay (BPP-LED) ${ }^{[46,50]}$ The shape of all gradient

(A)

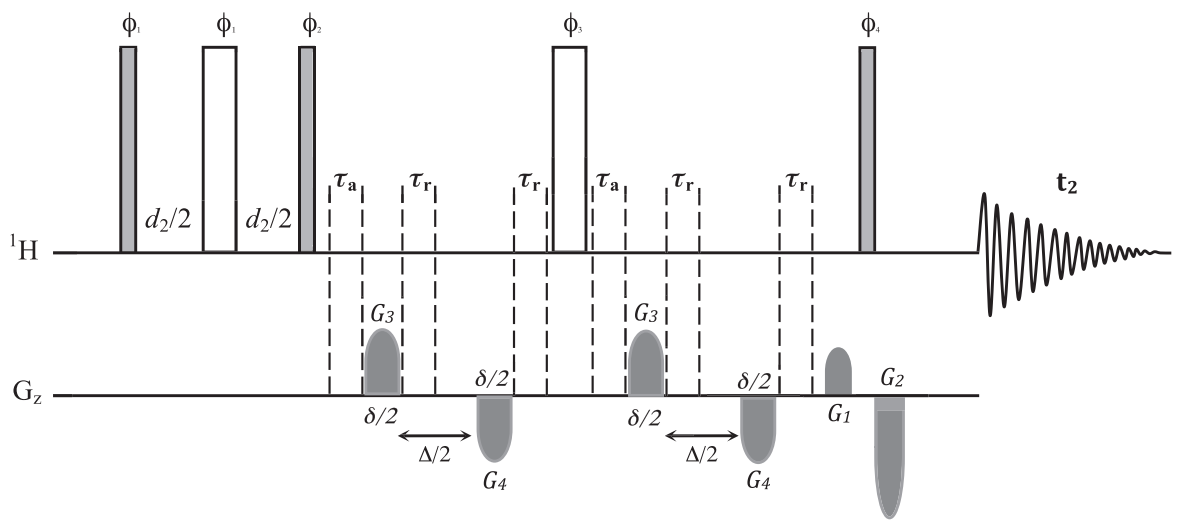

(B)

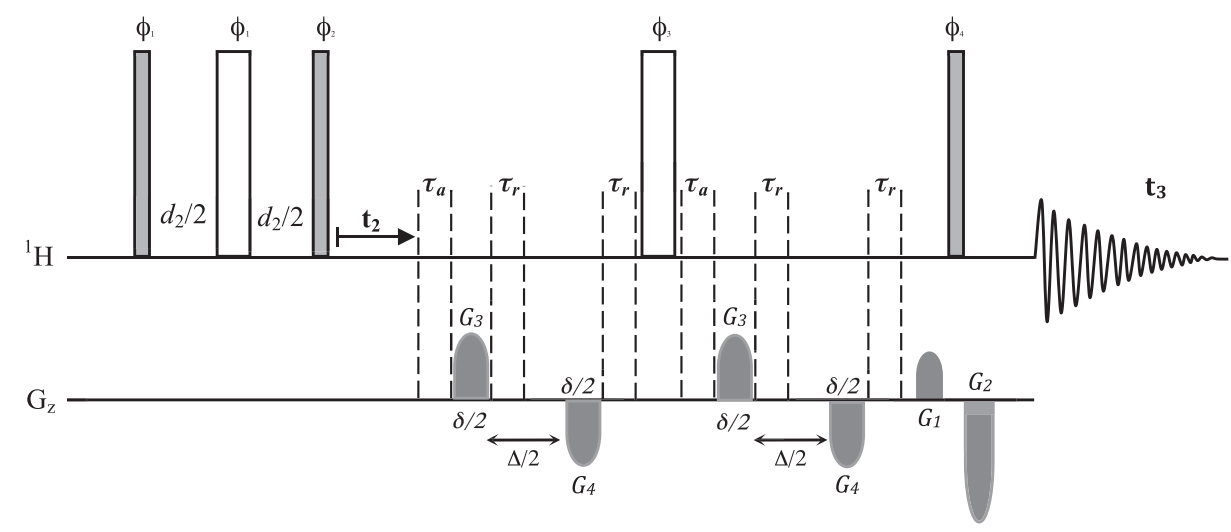

Figure 2. Convection compensated pulse sequences for $2 D(A)$ and 3D (B) MQDOSY-MQ. Black and white pulses represent rf pulse flip angles of $\pi / 2$ and $\pi$, respectively. The $\phi_{1}$ phase was equal to $\mathrm{x}$, while $\phi_{2}$ was chosen to select odd or even MQ orders ( $x$ or $y$ for even or odd order excitation, respectively). The duration of the preparation period $d_{2}$ was optimized for as uniform as possible excitation MQ of the desired coherence order (see text). The ratio of the coherence selection gradient pulses was chose to fulfill $G_{2}=p \times G_{1}$, where $p$ is the MQ coherence of choice. $\tau_{\mathrm{r}}$ are delays for gradient recovery times and $\tau_{\mathrm{a}}$ an additional delay equal to $\tau_{\mathbf{r}}$ introduced to center the second $\pi$ pulse. 
pulses was Smoothed Square and the LED delay was of $5 \mathrm{~ms}$. For all experiments, the diffusion delay $\Delta$ was set at $100 \mathrm{~ms}$, the gradient strength, $g$, was linearly incremented in 32 steps from $2 \%$ to $95 \%$ of its maximum value, and four scans were recorded for each experiment. For $\mathrm{MQ} / \mathrm{SQ}$ correlation schemes, MQ-coherences were excited with the standard sequence $90_{x}-d_{2} / 2-180_{x}-d_{2} / 2-90_{\phi}(\phi=x$ or $y$ for even or odd order excitation respectively) with coherence order selection by a pulse field gradient sandwich around the last $90^{\circ}$ pulse. ${ }^{[51]}$ The interval $d_{2}$ was first tentatively set to the inverse of half of the largest coupling of the spin systems and subsequently optimized by eye to obtain the highest and most uniform signal intensity along the series of molecules. MQ spectra were processed to produce absolute value plots. A reduced scale, divided by the quantum order, $p$, was used for the MQ dimension. This allows an easier comparison along different quantum orders. For the uniformly sampled (US) 3D MaxQDOSY-MaxQ, the indirect dimension was sampled by acquiring 256 increments. The Non-Uniformly Sampled version of the $3 \mathrm{D}$ experiment was acquired using a schedule automatically generated by the software TOPSPIN, followed by Compressed Sensing reconstruction. Compression factors of 4 and 8 were tested, in order to visually assess the conditions that provided no peak distortions.

\section{Results and Discussion}

\section{D MQDOSY:}

The $p$ QDOSY sequence (Fig. 2A) was used with parameters chosen to obtain a uniform overall diffusion-induced signal loss for all values of $p$ values explored. In practice, to compensate for the increased sensitivity to magnetic field gradients because of effective gyromagnetic ratios $(p \gamma)$ (see Eqn (1)), the gradient pulse duration $\delta$ was divided by $p$.

A crucial aspect for fine separation of mixture components using DOSY is compensation of convection effects. ${ }^{[47,48]}$ This nuisance is once again demonstrated here, for $p=4$ (Fig. 3). A standard Hahn spin-echo based 4Q-PGSE experiment shows significant convection effects, in the form of an extra modulation of the decay, and the diffusion based spin-echo attenuation failed to determine diffusion coefficients correctly. Indeed, in the presence of convection, the spin-echo attenuation is modulated by a cosine term and Eqn (1) becomes $^{[52-55]}$ :

$$
\cos \left(\gamma_{\text {eff }} \delta g v \Delta\right) e^{\left(-\gamma_{\text {eff }}^{2} g^{2} D \delta^{2}(\Delta-\delta / 3)\right)}
$$

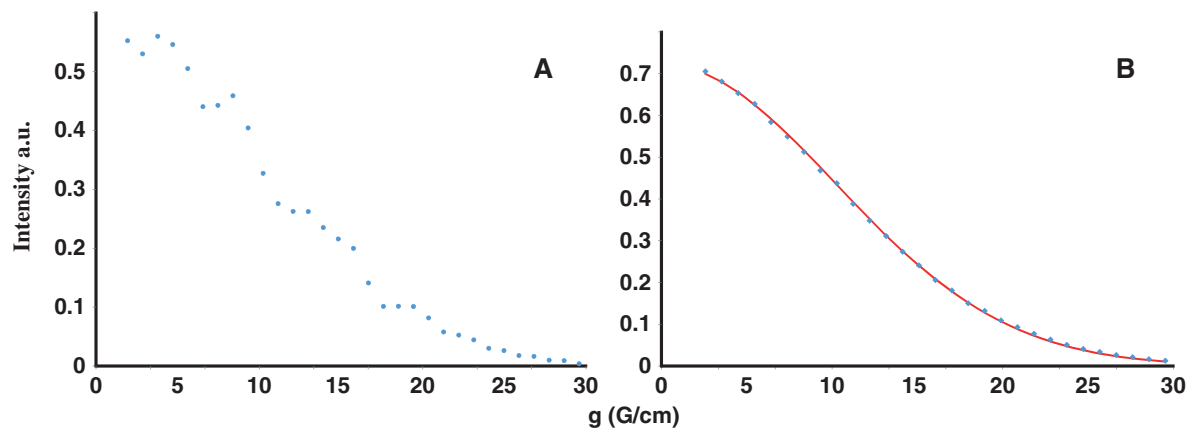

Figure 3. Diffusion decays corresponding to phenanthrene (peak at $8.8 \mathrm{ppm}$ ) obtained by the sequence of Fig. 2. A: with and B: without convection compensation, respectively. Dots: experimental data, Continuous curves: recalculated decay.
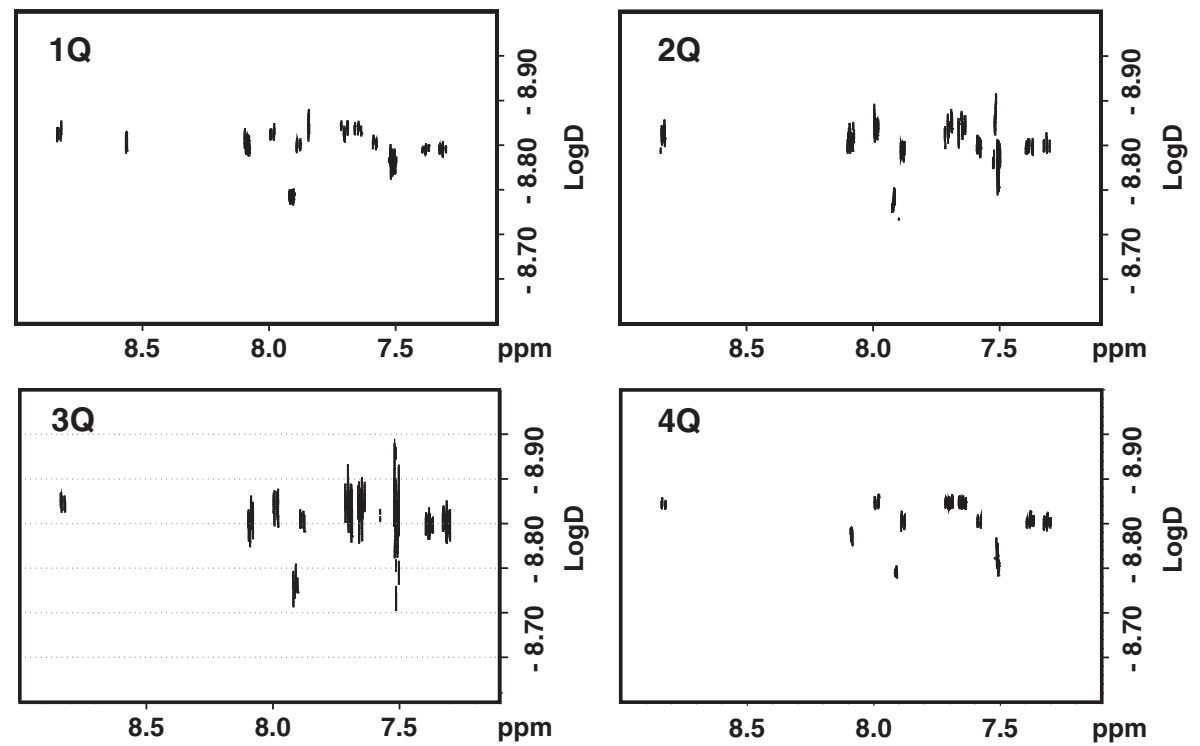

Figure 4. $p \mathrm{QDOSY}(p=1-4)$ experiments on a mixture of four aromatic compounds (anthracene, phenanthrene, naphthalene, and fluorene) obtained by the sequence of Fig. $2 \mathrm{~A}$. 
$v$ represents the velocity of convection. The other parameters are defined in Eqn (1).

Interestingly, in the same conditions of temperature control, by using a standard $1 \mathrm{Q}$ stimulated echo STE based PGSE, there was no observed convection effect (not shown). As shown in Eqn (2), convection effects appear amplified by the effective magnetogyric ratio $(p \gamma)$, and become a real nuisance, especially when highquantum orders ( $4 \mathrm{Q}$ in this study) are selected. The incorporation of a convection compensation scheme ${ }^{[56,57]}$ (Fig. 2) allowed recovering of a smooth diffusion attenuation curve.

The 2D pQDOSY (with $p=1-4$ ) spectra of the mixture are shown in Fig. 4. Neither 2D MQDOSY nor classic DOSY experiments were able to properly resolve all the peaks belonging to the four compounds, although the $p=4$ layout almost correctly split the signal, with a noticeable exception being the peaks around $7.52 \mathrm{ppm}$. Note that going to higher $p$ values did have a simplifying effect on the ${ }^{1} \mathrm{H}$ spectrum, although a modest one. In this specific case, just the peaks belonging to the central ring of anthracene and phenanthrene, bearing only two protons, disappear.

\section{D MaxQDOSY-MaxQ}

The pulse sequence of the 3D MQDOSY-MQ experiment is given in Fig. $2 \mathrm{~B}$, while the projections of the $3 \mathrm{D}$ pQDOSY-pQ $(p=4)$ spectrum of the analyzed mixture are shown in Fig. 5. Here 4QDOSY means that diffusion effect was encoded while the $4 Q$ coherence order was excited, which was also the MaxQ order for the four molecules in the mixture.

No ambiguity whatsoever in the molecular resolution is found in the case of the full 3D 4Q experiment. Here the projections along the three dimensions are all very well resolved and the MaxQ correlation (Fig. 5) provides the expected separation quality. On the other hand, the 4QDOSY-1Q projection plane is also better resolved than its 2D counterpart, with all peaks assigned to the correct spin system, without ambiguity. However, it is the 4 QDOSY-4Q projection plan that presents the most spectacular resolution, with only one peak per molecule located at the crossing of the value of the molecular diffusivity and on the average chemical shift, $\delta_{\text {MaxQ }}$. The separation of the four components is achieved in a straightforward way, and the diffusion coefficients are in agreement with those obtained by the standard STE based PGSE. The impressive amelioration in resolution in all dimensions is likely because of more efficient spread of the signal on a third dimension and to the specific properties of the MaxQ coherences. Overall, this reduces the overlap when performing the DOSY transform. In short, the fact that the MaxQ dimension presents no overlap profits not only to the MaxQDOSY-MaxQ projection, but also to the MaxQDOSY-1Q one.

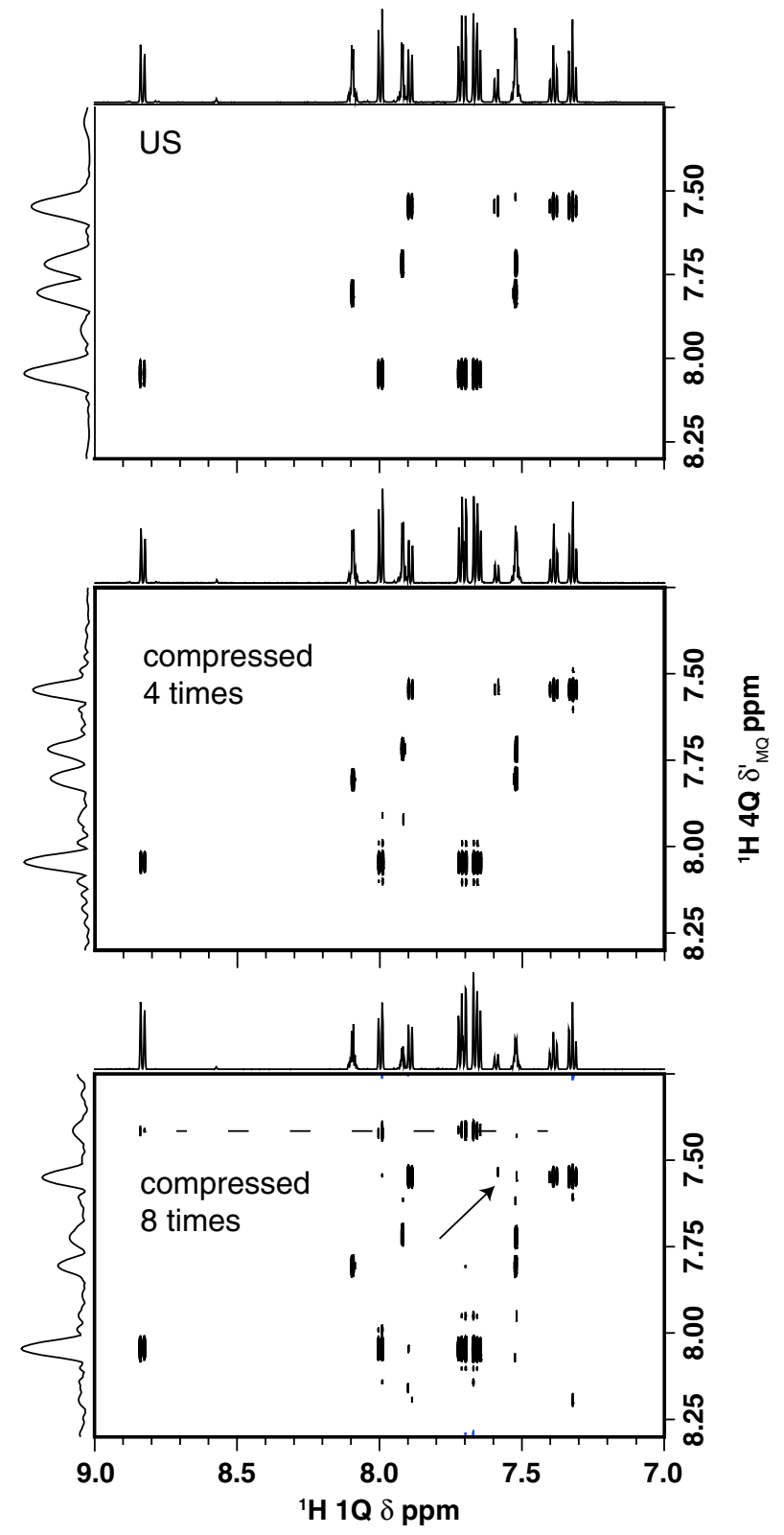

Figure 6. Comparison of $4 \mathrm{Q}-1 \mathrm{Q}$ spectra extracted from the $3 \mathrm{D} 4 \mathrm{QDOSY}-4 \mathrm{Q}$ experiments, corresponding to the first gradient strength (i.e. $2 \%$ of its maximum), recorded with Uniform Sampling US (top) and with non-uniform sampling (NUS), with a fourfold (middle) and eightfold compression rate (bottom). The arrow indicates the disappearing of a signal, while the broken line points out a replica artifact (see text).
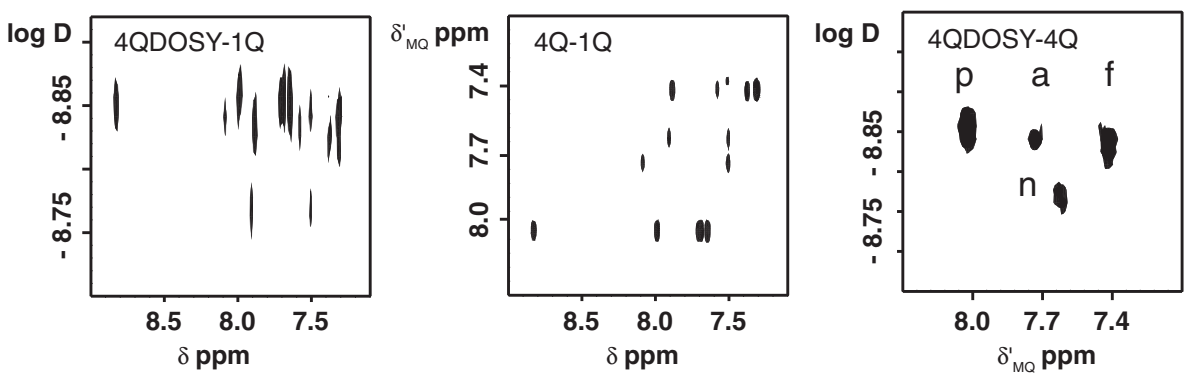

Figure 5. Projections of a $4 Q D O S Y-4 Q 3 D$ experiment. Left: $4 Q D O S Y-1 Q$ projection; middle: $4 Q-1 Q$ projection; right: $4 Q D O S Y-4 Q$ projection. The $4 Q$ spectrum was also the MaxQ order for the four molecules in the mixture (anthracene, phenanthrene, naphthalene, and fluorine). The scale in the $4 \mathrm{Q}$ dimension, $\delta_{\mathrm{MQ}}^{\prime}$, corresponds to a normalization to the $p$-quantum order (a division by 4 in this case, see text). 

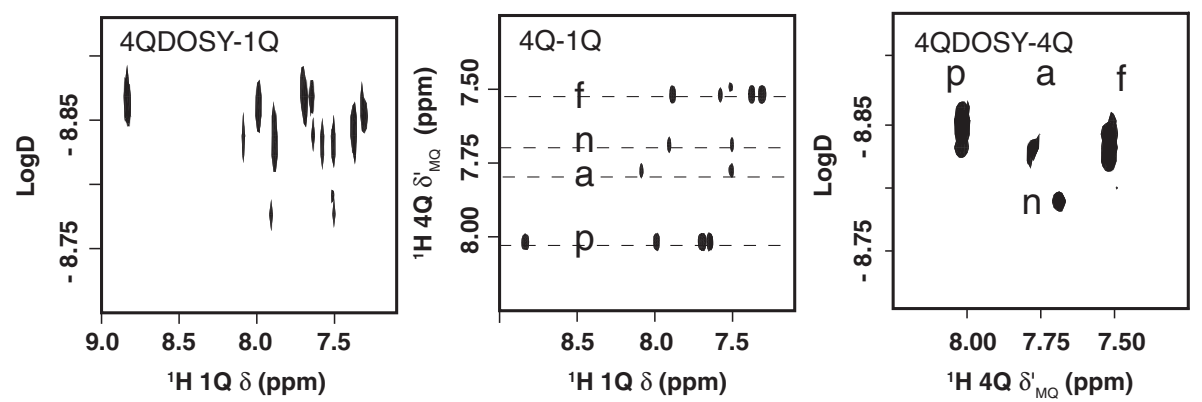

Figure 7. Projections of a 4QDOSY-4Q 3D experiment with a compression factor of eight. Left: $4 Q D O S Y-1 Q$ projection; middle: $4 Q-1 Q$ projection; right: $4 \mathrm{QDOSY}-4 \mathrm{Q}$ projection.

\begin{tabular}{|c|c|c|c|c|c|c|}
\hline Molecules & 2D 1QDOSY & 2D 2QDOSY & 2D 3QDOSY & 2D 4QDOSY & 3D 4Q-4QDOSY & NUS 3D 4Q-4QDOSY \\
\hline Fluorene & -8.853 & -8.853 & -8.855 & -8.855 & -8.845 & -8.840 \\
\hline Naphthalene & -8.795 & -8.795 & -8.783 & -8.798 & -8.780 & -8.784 \\
\hline Anthracene & -8.855 & -8.860 & -8.857 & -8.843 & -8.846 & -8.840 \\
\hline Phenanthrene & -8.869 & -8.873 & -8.875 & -8.875 & -8.865 & -8.870 \\
\hline
\end{tabular}

Again, all the processing was performed with standard commercial software, and more sophisticated algorithms for the DOSY transforms that have been demonstrated in the literature ${ }^{[58-61]}$ could further improve the analysis.

\section{Experiment time optimization by NUS}

The duration of 3D experiments represents a serious drawback. Indeed, in our case the duration of the pseudo-3D MaxQ-DOSY experiment was about $28 \mathrm{~h}$ ( 4 scans and 256 increments to sample the indirect MQ dimension). To minimize the experiment time without significant information loss, we applied the standard NUS approach available with the Topspin software. State-of-the-art understanding of the variables influencing a correct spectra reconstruction after NUS sampling is still very qualitative, and it is linked to the signalto-noise ratios. ${ }^{[62]}$ In the present case, we resorted to a visual assessment of a NUS parametrization capable of producing faithful spectra in a reasonable experimental duration. The compression rate that can be used without significant loss of resolution or of signals altogether, while avoiding processing artifacts, can be first inferred by analysis of Fig. 6 . For the sake of the analysis, the MaxQ-1Q spectra were investigated first. Here, they were actually the first planes of the full 3D experiment corresponding to a gradient value of $2 \%$ of the maximum. Comparison with the US spectra demonstrates that a factor of compression of eight introduces some reconstruction errors in the spectra, notably the disappearance of one peak of fluorene (shown by the arrow in the corresponding spectrum), and the appearance of shifted replicas of phenanthrene. Interestingly, such mistakes are not observed in the projection planes of the full 3D spectrum at the same level of compression (Fig. 7). This can be explained tentatively by the higher sparsity of the 3D dataset, which facilitates the reconstruction. In other words, the presence of artifacts could be different for each plane and thus average out.

The reduction in the time of the experiment pays a toll in overall sensitivity, and indeed resolution-enhanced NUS does not improve the $\mathrm{S} / \mathrm{N}$ ratio, which actually remains approximately of the same order of a US spectrum of the same length. Moreover, the nature of the noise is affected, with deviations from the typical Gaussian distribution. ${ }^{[62]} A$ theoretical or experimental study on the effect of such a variation on DOSY does not exist yet. However, a few general considerations can be drawn qualitatively. The NUS approach used here was chosen to compress the experimental time for constant resolution. Thus, the overall acquired signal would also be reduced. A reduction in $\mathrm{S} / \mathrm{N}$ in DOSY experiments may translate in a less precise determination of the diffusion coefficient. Such an effect is visible in comparing Figs. 5 and 7, where in this a small broadening of the lines in the projection planes sharing a $p Q D O S Y$ dimension can be observed. NUS schemes developed specifically for DOSY ${ }^{[63]}$ could be more adapted in this case and will be explored furthermore. Table 1 summarizes the measured diffusion properties for the four test molecules along the series of experiments. Non-overlapping signals were used in order to avoid interferences for other molecular systems. The reported values show a clear stability of the measure along the series of spectra. Even in the case of NUS, the somewhat broader peaks observed (Figs. 5 and 7) with respect to the US counterpart are not accompanied by a significant shift of the position of the peak maximum.

\section{Conclusions}

The combination of high-order MQ and DOSY can provide superior resolution for the analysis of mixtures. This can be understood as a combination of simplification of the ${ }^{1} \mathrm{H}$ spectrum because of MQ filtering on one hand and to the increased simplicity of the MQ spectra at high orders. Moreover, incorporation of the diffusion encoding on multiple quantum coherences allowed an increased sensitivity to magnetic field gradients. Thus, smaller gradients can be used and therefore smaller eddy-current or heating problems can be expected, within the limits of electronics linear response for very short PFG duration. On the other hand, thermal convection is also amplified and must be compensated for. The separation 
capability of the 3D MaxQDOSY-MaxQ experiment was demonstrated on a model mixture, sharing the same MaxQ order. Relevant properties of the experiment were clearly outlined, notably an improved performance of ILT algorithms for DOSY in the 3D setup, including a reduced number of artifacts in the NUS experiment. At any rate, improvement in the resolution is expected for general scenarios, on the lines of the MaxQ NMR setup. Finally, the experiment presented here, besides its obvious applicability for mixtures, could be devised for the characterization of slowly diffusing molecules, because of the apparent amplifying effect of the PFG intensity experienced by $p$-quantum coherences.

\section{References}

[1] E. O. Stejskal, J. E. Tanner. J. Chem. Phys. 1965, 42, 288-292. doi:10.1063/ 1.1695690 .

[2] J. E. Tanner. J. Chem. Phys. 1970, 52, 2523-2526. doi:10.1063/1.1673336.

[3] P. Stilbs. Prog. Nucl. Magn. Reson. Spectrosc. 1987, 19, 1-45. doi:10.1016/ 0079-6565(87)80007-9.

[4] P. Stilbs. Anal. Chem. 1981, 53, 2135-2137. doi:10.1021/ac00236a044.

[5] K. F. Morris, C. S. Johnson. J. Am. Chem. Soc. 1992, 114, 776-777. doi:10.1021/ja00028a063.

[6] K. F. Morris, C. S. Johnson. J. Am. Chem. Soc. 1992, 114, 3139-3141. doi:10.1021/ja00034a071.

[7] H. Barjat, G. A. Morris, S. Smart, A. G. Swanson, S. C. R. Williams. J. Magn. Reson. B 1995, 108, 170-172. doi:10.1006/jmrb.1995.1118.

[8] K. F. Morris, P. Stilbs, C. S. Johnson. Anal. Chem. 1994, 66, 211-215. doi:10.1021/ac00074a006

[9] E. K. Gozansky, D. G. Gorenstein. J. Magn. Reson. B 1996, 111, 94-96. doi:10.1006/jmrb.1996.0066

[10] D. Wu, A. Chen, J. C. S. Johnson. J. Magn. Reson., Ser. A 1996, 121, 88-91. doi:10.1006/jmra.1996.0142.

[11] S. Viel, S. Caldarelli. Chem. Commun. 2008, 2013-2015. doi:10.1039/ b802789g.

[12] M. Nilsson, A. M. Gil, I. Delgadillo, G. A. Morris. Anal. Chem. 2004, 76, 5418-5422. doi:10.1021/ac049174f.

[13] A. Tal, L. Frydman. Prog. Nucl. Magn. Reson. Spectrosc. 2010, 57, $241-$ 292. doi:10.1016/j.pnmrs.2010.04.001.

[14] A. L. Guennec, P. Giraudeau, S. Caldarelli, J.-N. Dumez. Chem. Commun. 2015, 51, 354-357. doi:10.1039/C4CC07232D.

[15] P. Giraudeau, E. Cahoreau, S. Massou, M. Pathan, J.-C. Portais, S. Akoka. ChemPhysChem 2012, 13, 3098-3101. doi:10.1002/cphc.201200255.

[16] M. Piotto, G. N. Manjunatha Reddy, S. Caldarelli. J. Magn. Reson. 2011 , 213, 107-111. doi:10.1016/j.jmr.2011.09.016.

[17] M. E. Zielinski, K. F. Morris. Magn. Reson. Chem. 2009, 47, 53-56. doi: $10.1002 / \mathrm{mrc} .2348$.

[18] J. S. Kavakka, V. Parviainen, K. Wähälä, I. Kilpeläinen, S. Heikkinen. Magn. Reson. Chem. 2010, 48, 777-781. doi:10.1002/mrc.2660.

[19] S. Viel, F. Ziarelli, S. Caldarelli. Proc. Natl. Acad. Sci. 2003, 100, 9696-9698. doi:10.1073/pnas.1533419100.

[20] G. Pages, C. Delaurent, S. Caldarelli. Anal. Chem. 2006, 78, 561-566. doi:10.1021/ac051454n.

[21] G. Pages, C. Delaurent, S. Caldarelli. Angew. Chem. Int. Ed. 2006, 45, 5950-5953. doi:10.1002/anie.200123456.

[22] G. N. M. Reddy, R. Ballesteros-Garrido, J. Lacour, S. Caldarelli. Angew. Chem. Int. Ed. 2013, 52, 3255-3258. doi:10.1002/anie.201209616.

[23] A. Wokaun, R. R. Ernst. Mol. Phys. 1978, 36, 317-341. doi:10.1080/ 00268977800101601.

[24] G. Drobny, A. Pines, S. Sinton, D. P. Weitekamp, D. Weemer. Faraday Symp. Chem. Soc. 1979, 13, 49.

[25] G. Bodenhausen. Prog. Nucl. Magn. Reson. Spectrosc. 1980, 14, 137-173. doi:10.1016/0079-6565(80)80007-0.

[26] T. J. Norwood. Prog. Nucl. Magn. Reson. Spectrosc. 1992, 24, 295-375. doi:10.1016/0079-6565(92)80005-Z.

[27] S. Sinton, A. Pines. Chem. Phys. Lett. 1980, 76, 263-267. doi:10.1016/ 0009-2614(80)87017-5.

[28] B. Baishya, G. N. M. Reddy, U. R. Prabhu, T. N. G. Row, N. Suryaprakash. J. Phys. Chem. A 2008, 112, 10526-10532. doi:10.1021/jp8055174.
[29] G. N. Manjunatha Reddy, T. N. Guru Row, N. Suryaprakash. J. Magn. Reson. 2009, 196, 119-126. doi:10.1016/j.jmr.2008.10.018.

[30] N. Suryaprakash, in Annual Reports on NMR Spectroscopy, (Ed: Graham A. W.), Academic Press, 2011, pp 61-110.

[31] S. Hebbar, U. R. Prabhu, N. Suryaprakash. J. Magn. Reson. 2012, 215, 2326. doi:10.1016/j.jmr.2011.12.008.

[32] G. N. Manjunatha Reddy, S. K. Nayak, T. N. Guru Row, N. Suryaprakash. Magn. Reson. Chem. 2009, 47, 684-692. doi:10.1002/mrc.2449.

[33] G. N. M. Reddy, S. Caldarelli. Magn. Reson. Chem. 2013, 51, 240-244. doi:10.1002/mrc.3938.

[34] G. N. M. Reddy, S. Caldarelli. Analyst 2012, 137, 741-746.

[35] G. N. M. Reddy, S. Caldarelli. Chem. Commun. 2011, 47, 4297-4299. doi:10.1039/c1cc10226e.

[36] G. N. M. Reddy, S. Caldarelli. Anal. Chem. 2010, 82, 3266-3269. doi:10.1021/ac100009y.

[37] R. R. Ernst, G. Bodenhausen, A. Wokaun, Principles of Nuclear Magnetic Resonance in One and Two Dimensions, 1991.

[38] S. S. Köcher, T. Heydenreich, Y. Zhang, G. N. M. Reddy, S. Caldarelli, H. Yuan, S. J. Glaser. J. Chem. Phys. 2016, 144, 164103. doi:10.1063/ 1.4945781.

[39] D. Zax, A. Pines. J. Chem. Phys. 1983, 78, 6333-6334. doi:10.1063/ 1.444559.

[40] J. F. Martin, L. S. Selwyn, R. R. Vold, R. L. Vold. J. Chem. Phys. 1982, 76, 2632-2634. doi:10.1063/1.443243.

[41] L. E. Kay, J. H. Prestegard. J. Magn. Reson. (1969) 1986, 67, 103-113. doi:10.1016/0022-2364(86)90413-0.

[42] B. E. Chapman, P. W. Kuchel. J. Magn. Reson., Ser. A 1993, 102, 105-109. doi:10.1006/jmra.1993.1075.

[43] L. Van Dam, B. Andreasson, L. Nordenskiöld. Chem. Phys. Lett. 1996, 262, 737-743. doi:10.1016/S0009-2614(96)01160-8.

[44] C. H. Sotak. J. Magn. Reson. (1969) 1990, 90, 198-204. doi:10.1016/00222364(90)90379-N.

[45] C. Dalvit, J. M. Böhlen. NMR Biomed. 1997, 10, 285-291. doi:10.1002/ (SICI)1099-1492(199709)10:6<285::AID-NBM485>3.0.CO;2-1.

[46] M. Shukla, K. Dorai. Appl Magn Reson 2012, 43, 485-497. doi:10.1007/ s00723-012-0316-y.

[47] A. Jerschow, N. Müller. J. Magn. Reson. 1997, 125, 372-375. doi:10.1006/ jmre.1997.1123.

[48] I. Swan, M. Reid, P. W. A. Howe, M. A. Connell, M. Nilsson, M. A. Moore G. A. Morris. J. Magn. Reson. 2015, 252, 120-129. doi:10.1016/j. jmr.2014.12.006.

[49] K. Kazimierczuk, V. Y. Orekhov. Angew. Chem. Int. Ed. 2011, 50, 55565559. doi:10.1002/anie.201100370.

[50] D. H. Wu, A. D. Chen, C. S. Johnson. J. Magn. Reson., Ser. A 1995, 115 260-264. doi:10.1006/jmra.1995.1176.

[51] A. Bax, P. G. De Jong, A. F. Mehlkopf, J. Smidt. Chem. Phys. Lett. 1980, 69, 567-570. doi:10.1016/0009-2614(80)85130-X.

[52] N. Hedin, T. Y. Yu, I. Furó. Langmuir 2000, 16, 7548-7550. doi:10.1021/ la000595b.

[53] B. Antalek. Concepts Magn. Reson. 2002, 14, 225-258. doi:10.1002/ cmr.10026.

[54] A. Jerschow, N. Müller. J. Magn. Reson. 1998, 132, 13-18. doi:10.1006/ jmre.1998.1400.

[55] G. Zheng, W. Price. J. Biomol. NMR 2009, 45, 295-299. doi:10.1007/ s10858-009-9367-2.

[56] M. Nilsson, G. A. Morris. J. Magn. Reson. 2005, 177, 203-211. doi:10.1016/j.jmr.2005.07.019.

[57] K. I. Momot, P. W. Kuchel. J. Magn. Reson. 2005, 174, 229-236. doi:10.1016/j.jmr.2005.02.003.

[58] M. Nilsson. J. Magn. Reson. 2009, 200, 296-302. doi:10.1016/j. jmr.2009.07.022.

[59] C. D. Eads, I. Noda. J. Am. Chem. Soc. 2002, 124, 1111-1118. doi:10.1021/ ja011819v.

[60] R. Huo, R. Wehrens, J. V. Duynhoven, L. M. C. Buydens. Anal. Chim. Acta 2003, 490, 231-251. doi:10.1016/S0003-2670(03) 00752-9.

[61] I. Toumi, S. Caldarelli, B. Torrésani. Prog. Nucl. Magn. Reson. Spectrosc. 2014, 81, 37-64. doi:10.1016/j.pnmrs.2014.06.002.

[62] A. Le Guennec, J.-N. Dumez, P. Giraudeau, S. Caldarelli. Magn. Reson. Chem. 2015, 53, 913-920. doi:10.1002/mrc.4258.

[63] M. Urbańczyk, W. Koźmiński, K. Kazimierczuk. Angew. Chem. 2014, 126, 6582-6585. doi:10.1002/ange.201402049. 